Archives de sciences sociales des religions

154 | Avril-juin 2011

Varia

\title{
Du don au paiement rituel
}

L'échange en question

Raymond Jamous

\section{(2) OpenEdition}

Journals

Édition électronique

URL : http://journals.openedition.org/assr/23186

DOI : $10.4000 /$ assr.23186

ISSN : $1777-5825$

Éditeur

Éditions de l'EHESS

Édition imprimée

Date de publication : 1 avril 2011

ISBN : 978-2-7132-2302-0

ISSN : 0335-5985

Référence électronique

Raymond Jamous, "Du don au paiement rituel », Archives de sciences sociales des religions [En ligne], 154 | Avril-juin 2011, mis en ligne le 01 août 2011, consulté le 30 avril 2019. URL : http:// journals.openedition.org/assr/23186 ; DOI : 10.4000/assr.23186 


\section{Raymond Jamous}

\section{Du don au paiement rituel L'échange en question ${ }^{1}$}

Il n'est pas rare dans les sociétés indiennes comme dans les textes brahmaniques, de considérer le mariage de la manière suivante : "Un père trouve ou agrée un fiancé et lui donne sa fille. Le terme kanyadana ("don de la jeune fille”) qui désigne une phase particulière de la cérémonie, en révèle aussi le sens général. Le père donne, il ne vend pas, il n'échange pas » (Malamoud, 1974-1975 : 125). Dans cette optique, le don n'est pas un échange et il doit être considéré comme un fait rituel. Par ailleurs, Charles Malamoud souligne qu'offrir un don rituel peut être comparé à une offrande sacrificielle. Ce sont ces propositions que nous voulons examiner à l'aide des approches qui associent étroitement don et échange et en nous interrogeant sur le don orienté sans retour possible et ses implications - dans les cérémonies de mariage à partir d'un cas ethnographique.

\section{Donner n'est pas échanger}

\section{Échange et don chez Marcel Mauss}

Dans son Essai sur le don, Marcel Mauss associe étroitement don et échange, avec la triade : donner, recevoir, rendre. Nous ne citerons que deux passages où le glissement du don à l'échange est manifeste. Après avoir souligné « le caractère volontaire, pour ainsi dire, apparemment libre et gratuit ; et cependant contraint et intéressé de ces prestations " (1960:147), Mauss ne traitera que d'une question : "Quelle est la règle de droit et d'intérêt qui, dans les sociétés de type arriéré ou archaïque, fait que le présent reçu est obligatoirement rendu ?» (ibid.: 148). Plus loin, Mauss précise sa perspective : «D'abord ce ne sont pas des individus, ce sont des collectivités qui s'obligent mutuellement ; échangent et contractent ; les personnes présentes au contrat sont des personnes morales : clans, tribus, familles qui s'affrontent et s'opposent soit en groupes se faisant face sur le terrain même, soit par l'intermédiaire de leurs chefs, soit de ces deux façons à la fois.

1. Je voudrais remercier ma femme Marie-Jo Jamous, Laurence Caillet, Charles Malamoud, Anne-Christine Taylor et Romain Simenel pour une lecture attentive de ce texte, pour leur encouragement et pour leurs remarques qui ont permis de préciser et d'affiner le propos. 
De plus, ce qu'ils échangent, ce n'est pas exclusivement des biens et des richesses, des meubles et des immeubles, des choses utiles économiquement. Ce sont avant tout des politesses, des festins, des rites, des services militaires, des femmes, des enfants, des danses, des fêtes, des foires dont le marché n'est qu'un des moments et où la circulation n'est qu'un des termes d'un contrat beaucoup plus général et beaucoup plus permanent (ibid. : 150-151).

Il ne s'agit pas de revenir sur tout le développement que propose Mauss dans l'Essai sur le don, mais de signaler une difficulté concernant l'équivalence établie entre don et échange. À propos du droit hindou dans lequel la notion de dan correspond au don, Mauss insiste sur l'obligation de donner et celle de recevoir, mais il n'est pas question d'une quelconque obligation de rendre. Plus même, il indique dans la note 1, page 243 : «Il faut convenir que, sur le sujet principal de notre démonstration, l'obligation de rendre, nous avons trouvé peu de faits dans le droit hindou... Même le plus clair consiste dans la règle qui l'interdit ». Certes la note continue pour montrer comment dans les cérémonies funéraires, on peut se rapprocher de donner et rendre. On dit bien que le don produira ses effets dans une autre vie pour le donateur, mais cela reste vague comme l'idée du mérite après la mort. Qu'est-ce à dire, sinon qu'il peut y avoir don sans qu'il y ait échange. Il ne s'agit pas de remettre en question le propos de M. Mauss mais de souligner que la triade donner, recevoir, rendre ne peut être généralisée et que le droit hindou nous conduit à la question : Qu'est-ce que le don quand il n'y a pas d'obligation de rendre ou même quand il est interdit de rendre ? ${ }^{2}$

\section{Échange matrimonial et alliance de mariage}

Cette étroite association entre don et échange se retrouve dans les deux approches du mariage par Claude Lévi-Strauss et Louis Dumont. Dans son introduction à Sociologie et Anthropologie de Mauss, Lévi-Strauss a insisté à ce sujet sur le caractère synthétique du don en notant : «(...) c'est l'échange qui constitue le phénomène primitif et non les opérations discrètes en lesquelles la vie sociale le décompose » (1960: XXXVIII). La notion d'échange devient première dans Les structures élémentaires de la parenté où après avoir montré que la prohibition de l'inceste constitue la face négative de l'échange, Lévi-Strauss poursuit :

«En quoi consistent les structures mentales auxquelles nous avons fait appel et dont nous croyons pouvoir établir l'universalité ? Elles sont, semble-t-il, au nombre de trois : l'exigence de la Règle comme Règle ; la notion de réciprocité comme la forme la plus immédiate sous laquelle puisse être intégrée l'opposition de moi et d'autrui, enfin la caractère synthétique du Don, c'est-à-dire le fait que le transfert consenti d'une valeur d'un individu à un autre change ceux-ci en partenaires, et ajoute une qualité nouvelle à la valeur transférée. » (1967: 108-109)

2. Pour une critique du don selon une autre perspective que celle que nous adoptons ici, voir Alain Testard, 2007. 
Lévi-Strauss considère deux modes d'échanges : l'échange restreint dont le prototype est le mariage entre cousins croisés bilatéraux, l'échange généralisé dont le prototype est le mariage avec la cousine croisée matrilatérale et dans une moindre mesure le mariage avec la cousine croisée patrilatérale. Pour Mauss, l'échange des femmes est une des formes parmi d'autres que prend «le système des prestations totales ». Lévi-Strauss reprend cette idée quand il écrit que, dans l'échange, il s'agit de : "prestations totales, dont le mariage offre un exemple, en même temps qu'il en fournit l'occasion. Ces prestations totales portent... sur des biens matériels, sur des valeurs sociales telles que privilèges, droits et obligations, et sur des femmes » (ibid. : 148). Mais dans la conclusion de l'ouvrage, il revient sur ce sujet et après avoir noté que l'exogamie est un élément important de "l'intégration des unités partielles ", il souligne: "Il n'est pas exagéré de dire qu'elle [l'exogamie] est l'archétype de toutes les autres manifestations à base de réciprocité, qu'elle fournit la règle fondamentale et immuable qui assure l'existence du groupe comme groupe " (ibid. : 551). Il conclut à ce sujet : "Parce que le mariage est échange, parce que le mariage est archétype de l'échange, l'analyse de l'échange peut aider à comprendre cette solidarité qui unit le don et le contredon, le mariage aux autres mariages " (ibid. : 554).

Louis Dumont reprend la double proposition de Lévi-Strauss : l'échange de mariage et la centralité du mariage par rapport aux autres types de prestations, en modifiant la première et en précisant la seconde. Il va distinguer chez LéviStrauss, deux théories :

- une théorie générale de la parenté, "centrée sur une interprétation structurale de la prohibition de l'inceste » (1971 : 117), laquelle « (...) est l'expression négative d'une loi d'échange, l'expression partielle d'un principe universel de réciprocité, la contrepartie nécessaire d'une instauration de liens sociaux entre familles » (ibid. : 117);

- une théorie restreinte qui "porte seulement sur des sociétés d'un certain type, des sociétés qui possèdent des règles positives relatives au choix du conjoint dans le mariage du point de vue de la parenté. Il faudrait même dire des règles positives d'un certain type, puisque ces sociétés prescrivent ou préfèrent uniformément le mariage entre personnes tombant dans la catégorie des "cousins croisés" » (ibid. : 116). C'est à ce propos et à ce propos seulement que Dumont utilise « la théorie de l'alliance de mariage " (ibid. : 116).

Louis Dumont va ajouter deux principes supplémentaires à cette théorie restreinte : le caractère répétitif et diachronique des alliances matrimoniales et l'opposition entre deux catégories complémentaires : la consanguinité (ceux avec qui le mariage est interdit) et l'affinité (ceux avec qui on se marie selon les règles positives), d'où l'importance de l'analyse terminologique. L'aspect échange passe au second plan : "Les règles de mariage ont donc des implications structurales infiniment plus larges que le seul arrangement des groupes. Par opposition aux expressions fondées sur l'échange, etc., l'expression d'alliance de mariage peut 
couvrir à la fois l'aspect général des implications mentales et l'aspect particulier des implications de morphologie sociale... L'expression suggère surtout, ce qui est important du point de vue conceptuel, mais aussi pour les implications morphologiques, que l'affinité est dans ces systèmes quelque chose de permanent, quelque chose qui s'hérite ou se transmet d'une génération à l'autre comme nous avons accoutumé de le penser de la seule consanguinité »(ibid.: 134). Pour Dumont, il y a donc bien plus dans l'alliance de mariage que de l'échange, mais il ne remet pas en cause la validité de celui-ci et ne différencie pas nettement le don de l'échange. Cela dit, la discussion à propos du mariage avec la cousine croisée matrilatérale indique bien que l'échange fait problème. Si la règle positive ne fait que distinguer entre un preneur et un donneur, sans proposer aucune idée de cycle ou de bouclage, comment établir ce dernier en prenant des exemples empiriques qui échappent à la conscience des intéressés ? Mélanger dans une même construction du modèle les règles et la pratique ne peut qu'être source de confusion. C'est dans ce sens qu'il faut comprendre une remarque importante de LéviStrauss dans La Pensée samvage: "Nous avons paru trop souvent être à la recherche d'une genèse inconsciente de l'échange matrimonial... il aurait fallu distinguer davantage entre l'échange... (fait de praxis) et les règles conscientes... par le moyen desquelles ces mêmes groupes s'emploient à le codifier et à le contrôler. S'il y a un enseignement à tirer des enquêtes ethnographiques de ces vingt dernières années, c'est que cet aspect est beaucoup plus important que les observateurs - victimes en cela de la même illusion que Sartre - ne l'avaient généralement soupçonné » (1962 : 333). Louis Dumont reprenant cette révision des Structures élémentaires de la parenté ajoute : "En d'autres termes, il n'y a pas d'explication de l'empirique qui ne passe par l'idéologique. Autrement dit, le Lévi-Strauss des Structures est en fin de compte préstructuraliste » (1971 : 132).

C'est dans sa monographie Une sous-caste en Inde du sud que cet anthropologue se rapproche le plus d'une formulation qui permet de distinguer entre don et échange. Chez les Pramallai Kallar de l'Inde du Sud, le mariage avec la cousine croisée matrilatérale permet de distinguer le preneur du donneur de femme sans faire intervenir la notion de fermeture d'un cycle, de bouclage. Dans ce contexte, selon Dumont, l'oncle maternel n'est pas un consanguin (le frère de la mère), mais un affin (le beau-frère du père) et il est potentiellement le beau-père de son neveu utérin. De ce point de vue, l'affinité est transgénérationnelle. C'est dans ce contexte que Dumont insiste sur l'articulation entre mariage asymétrique et prestations orientées de l'oncle maternel au neveu utérin. Voilà comment il conclut le chapitre consacré à ce dernier aspect :

«On peut définir les caractères particuliers de la relation : oncle maternel-neveu utérin, par rapport aux autres relations d'alliance. Elle diffère de la relation entre cousins croisés, en ce qu'elle inclut une différence de génération. Ceci n'altère pas sa nature fondamentale, mais c'est important parce que la relation d'alliance devient ici non seulement asymétrique, mais projective, prend une dimension diachronique. Le fait qu'il existe une certaine transmission des biens sous forme de dons, le fait que si le 
beau-père lui aussi donne, c'est seulement l'oncle maternel qui a des fonctions cérémonielles, tout cela suggère que le rôle de l'oncle maternel, s'il est fondé sur l'alliance, doit son importance à ce qu'il contient l'alliance sous une forme généalogique, c'està-dire non seulement l'alliance sous sa forme synchronique... mais l'alliance en même temps que sa transmission. » $(1957: 263)$

Le mariage asymétrique et les prestations orientées de l'oncle maternel s'inscrivent dans l'affinité comprise dans sa dimension diachronique, On est toujours dans l'orientation et non dans l'échange.

\section{L'esprit du don : du hau au poison, une interprétation à revoir}

À ce stade de l'analyse, il est important de souligner le mérite de Jonathan Parry qui a été l'un des rares à insister sur le fait que le don indien, le dan, est orienté sans rendu possible. Mais cet anthropologue britannique spécialiste de l'Inde veut aller plus loin en essayant d'expliquer le pourquoi de cette nonréciprocité. S'inscrivant dans la lignée de Mauss, c'est la référence à «l'esprit du don ", ou plus précisément à la relation étroite entre le sujet donateur et l'objet donné qui lui permet d'expliquer à la fois l'échange et le refus de l'échange. C'est à propos du hau que cette notion de l'esprit du don est introduite par Mauss. Ce sujet ayant été largement discuté dans la littérature anthropologique, il ne s'agit pas d'y revenir, mais de noter comment Mauss interprète le hau :

"Ce qui, dans le cadeau reçu, échangé, oblige, c’est que la chose reçue n'est pas inerte. Même abandonnée par le donateur, elle est encore quelque chose de lui. Par elle, il a prise sur le bénéficiaire, comme par elle, propriétaire, il a prise sur le voleur. Car le taonga est animé du hau de sa forêt, de son terroir, de son sol; il est vraiment "native" : le hau poursuit tout détenteur. Il poursuit non seulement le premier donataire, même éventuellement un tiers, mais tout individu auquel le taonga est simplement transmis. Au fond, c'est le hau qui veut revenir au lieu de sa naissance, au sanctuaire de la forêt et du clan et au propriétaire. » (1960: 159-160)

Pour J. Parry, cet esprit du don existe dans le dan indien. Reprenant la discussion autour de l'analyse du hau maori, il indique que cette relation étroite entre sujet donateur et objet donné est un fait inscrit dans la circulation des prestations et permet de comprendre la réciprocité et le retour du don. On retrouve le même type de rapport sujet - objet dans le don indien sauf qu'ici il y a un refus de la réciprocité. J. Parry retrouve des indices dans le texte de Mauss (voir ci-dessous). Il signale, en reprenant son travail sur Bénarès ${ }^{3}$ et en s'appuyant sur celui de

3. Voilà ce qu'écrit J. Parry: "I have described, for example, how the Brahman priests of Benares see themselves as endlessly accumulating the sin they accept with the gifts of the pilgrims and mourners who visit the city, and how they liken themselves to a sewer through which the moral filth of their patrons is passed. Theoretically they should be able to 'digest' the sin by dint of various ritual procedures of expiation, and by donating the gifts they receive to another Brahman with increment. But quite apart from the fact that this is plainly an economic impossibility, they sadly admit ignorance of the correct ritual procedures. The sewer becomes a cesspit, with the result that the priest contracts leprosy and rots; he dies a terrible and premature death and then faces the torments of hell” (1986: 60). Voir aussi Parry, 1994. 
G. Raheja ${ }^{4}$, que, dans le monde indien, il existe aussi une association étroite entre un sujet donné et l'objet transmis, mais cette relation est considérée comme néfaste, dangereuse pour celui qui possède l'objet à donner et comme un poison pour celui qui le reçoit et qui doit essayer de se débarrasser de sa dangerosité par des rituels appropriés. Il souligne aussi que le don est un substitut du sacrifice : de la même manière que le rituel établit une relation étroite entre le sacrifiant et la victime qui sera offerte, avant de les séparer par l'acte de tuer cette dernière, offrir un don, c'est à la fois donner une part de sa personne et marquer la distance avec celui à qui l'on cède ce bien. La chose donnée doit être aliénée et ne pas être rendue. Voici comment J. Parry conclut son interprétation :

"There is no question, then, of the gift being a loan or pledge. It is alienated in an absolute way, and the very definition of the gift is that it involves the complete extinction of the donor's proprietary rights in favor of the recipient (Aiyar, 1941 : 77 ; Law 1926 : i). The gift threatens to cement the two together in a dangerous interdependence; but every attempt is made to sever their bond by insisting on the complete alienation of the thing. Under no circumstances, and on pain of terrible supernatural penalties, is the gift resumed. Its evil "spirit" must not come back. While Mauss originally introduced this notion of "spirit" explain the inalienability of the object and the necessity of making a return, what it in fact explains in this context is why the gift must be alienated, should never return, and should endlessly be handed on. " (1986: 461)

En bref, le don orienté possède un « esprit du mal » et la propriété du bien doit être cédée au donataire sans qu'il soit possible à ce dernier de le rendre.

Cette analyse soulève de nombreuses difficultés. Tout d'abord, il est difficile de généraliser l'idée que le don est un poison et de ne voir dans cette prestation que son aspect dangereux. La vache offerte comme don à un brahmane a une étincelle divine et n'est pas simplement un poison. Une femme donnée en mariage est un bienfait pour ceux qui le reçoivent car elle va enfanter, permettre la continuation de la lignée. Souligner que le don est un substitut du sacrifice est une idée intéressante, mais l'analogie ne va pas dans le sens de l'interprétation faite par J. Parry : le sacrifiant comme la victime sont consacrés, et la victime ne peut être offerte que parce qu'elle contient une forme de pureté, de divinité en elle. Il s'agit d'établir un lien et non simplement de séparer. Si le don est donc le poison que l'on offre, et qu'il faut continuer à le donner et de l'autre côté à le recevoir, on peut se demander pourquoi la société s'installe dans un tel enchaînement d'actes aux effets négatifs.

Mais la critique la plus importante que l'on peut faire à cette interprétation, concerne ce qu'elle retient de Mauss, c'est-à-dire "l'esprit du don ", la relation entre le sujet donateur et l'objet donné. Ce qui est mis à l'avant-plan pour le

4. J. Parry résume le point de vue de Raheja de la manière suivante : "Such gifts 'send away' inauspiciousness from the donor to the recipient, who may be a Brahman, Barber, Sweeper or a wife-taking affine, to whom the gift will bring misfortune unless the correct ritual precautions are taken” (1986: 460). Voir Raheja, 1988. 
hau comme pour le dan indien, c'est la relation de la personne à l'objet et la relation sociale entre les partenaires impliqués par cette circulation des dons passe au second plan. Mauss qui a bien vu ce problème, a certes signalé que le rituel établit un rapport entre le sujet donateur et la chose donnée :

\begin{abstract}
"Nulle part la liaison entre la chose donnée et le donateur, entre la propriété et le propriétaire n'est plus étroite que dans les règles concernant le don de la vache. Elles sont illustres. En les observant, en se nourrissant d'orge et de bouse de vache, en se couchant à terre, le roi Dharma (la loi), Yudhisthira lui-même, le héros principal de l'épopée, devint un "taureau" entre les rois. Pendant trois jours et trois nuits, le propriétaire de la vache l'imite et observe le "vœu qui vit de dons, prétend les refuser". Il se nourrit exclusivement des "sucs de la vache" : eau, bouse, urine pendant une nuit sur trois... Pendant une nuit sur trois, il couche avec les vaches, sur le sol comme elles, et ajoute le commentateur "sans se gratter, sans tracasser la vermine", s'identifiant ainsi “en âme unique à elles”." (Mauss, 1960 : 247)
\end{abstract}

Il ajoute que le don peut être dangereux, mais son appréciation à ce sujet est différente de celle de J. Parry ou de G. Raheja :

"Le don est donc à la fois ce qu'il faut faire, ce qu'il faut recevoir et ce qui est cependant dangereux à prendre. C'est que la chose donnée elle-même forme un lien bilatéral et irrévocable, surtout quand c'est un don de nourriture. Le donataire dépend de la colère du donateur, et même chacun dépend de l'autre. Aussi ne doit-on pas manger chez son ennemi. » (Mauss, $1960: 249$ )

Plus que la dangerosité en soi, tout est dans la manière de gérer la relation entre les partenaires. Il n'en reste moins que l'analyse de J. Parry mérite d'être prolongée sur deux points : l'orientation du don et le rapprochement qu'il fait entre don et sacrifice, comme on le verra plus bas dans le cas ethnographique des Meo.

$\mathrm{Si}$, au terme de cette première partie, l'on synthétise les analyses du don dans l'institution matrimoniale, on se trouve devant trois propositions: pour LéviStrauss, donner sa sœur en mariage conduit à l'échange, pour Dumont le mariage d'une sœur en Inde du Sud introduit la relation d'affinité entre beaux-frères et pour Parry, toujours pour l'Inde, il y a transfert des droits sur l'objet donné, en la circonstance la fille mariée qui cesse d'être membre de son groupe d'origine pour appartenir dorénavant à celui de son mari. Le don n'est plus que le moyen de l'échange, de l'affinité ou du transfert de propriété et dans le mariage indien, la fille donnée n'est pas un acteur, mais un moyen, un instrument de la relation ou de l'affirmation d'un groupe par rapport à l'autre. En fait, on peut se demander s'il ne faut pas complexifier les faits et considérer que la personne donnée n'est pas un objet inerte, mais se transforme dans le mouvement qui la fait passer de son groupe d'origine à celui de son mari. À la différence de ce dernier qui s'inscrit dans la lignée où il est né, peut-on dire que la femme mariée est simplement transférée ou bien qu'elle est marquée par la double appartenance, celui de son groupe d'origine et celui où elle a été donnée ? N'est-elle pas la seule à conjuguer, associer consanguinité et affinité, plus encore transcender cette distinction ? L'exemple des Meo de l'Inde du Nord va nous servir pour tester cette 
nouvelle analyse du don orienté de la femme et de ses implications ${ }^{5}$. Mais pour cela, il faudra considérer comment la parenté se construit dans les rites, notamment ceux du mariage.

\section{Du don rituel au paiement sacrificiel}

Les Meo forment une caste de guerriers Rajput et une communauté musulmane endogame installée dans plus de mille villages, au Rajasthan essentiellement, constituant une population de trois cent mille personnes vivant parmi d'autres castes, la plupart hindoues et d'autres musulmanes. Ils sont répartis en un certain nombre de clans patrilinéaires exogames, certains associés à un territoire, d'autres répartis dans le territoire des autres. Dans cette caste, l'échange et le don orienté sans retour possible relèvent respectivement de deux classes de parents opposés et complémentaires : les consanguins et les affins. C'est durant les cérémonies de mariage qu'ils se déroulent.

\section{Échange et dette : relations entre consanguins}

Les consanguins ${ }^{6}$ du côté du marié comme du côté de la mariée apportent une prestation en argent qui doit être rendue au donateur à l'occasion d'une cérémonie qui se tiendra chez lui. Il s'agit donc bien ici d'un échange. En fait, tout échange entre consanguins est pensé aussi en termes de dette. La prestation peut être ici de 5, 11, 21, 51 ou 101 roupies. Elle est composée de trois parties. Prenons l'exemple de 51 roupies données par A à B : 25 sont considérées comme le remboursement d'une dette de A envers B établie lors d'un précédent mariage, d'une circoncision, etc., 25 établissent une relation de dette de B envers A à rembourser dans le futur. Le 1 marque le moment présent. Cette prestation d'un consanguin est appelée nota. Ce terme est utilisé aussi dans un autre contexte du mariage. Les parents du marié ou de la mariée envoient un membre d'une caste de service (un barbier) inviter les consanguins aux cérémonies du mariage.

5. Les données sur cette communauté sont reprises et réinterprétées de mon ouvrage : La relation frère-sœur, parenté et rites chez les Meo de l'Inde du Nord, 1991.

6. Dans la littérature anthropologique, le terme de consanguinité a pris deux sens : le premier indique tous ceux avec qui on est lié par le sang que ce soit en termes de germanité ou de filiation. Dans ce contexte, rien n'est dit sur le mariage puisqu'on peut trouver des cas où l'on se marie dans la consanguinité (notamment dans les sociétés où il existe des mariages avec la cousine parallèle patrilatérale) et d'autres qui l'interdisent. Le second sens, que nous retenons ici, et que L. Dumont a désigné comme «la consanguinité terminologique » définit tous les parents avec qui le mariage est interdit. De ce point de vue, la consanguinité s'oppose à l'affinité. Il existe des parents par consanguinité comme des parents par affinité. Dans le cas où il existe un mariage de cousins croisés, ceux-ci sont à considérer comme des affins par opposition aux cousins parallèles. Plus encore, comme l'a souligné Dumont, l'oncle maternel peut être considéré comme affin de deux manières : comme le beau-frère du père et quand il donne sa fille en mariage à son neveu utérin, comme beau-père de son gendre. 
Ce délégué transmet l'invitation et doit donner une pièce de 1 roupie pour souligner qu'il a bien fait son travail et que l'invité a accepté de venir à la fête. Cette remise d'une petite somme qui marque l'acte d'invitation, doit être rapprochée du 1 lors de la remise de la prestation. Cette prestation de 1 roupie est le sceau de la relation, du lien inscrit dans une temporalité indéfinie. Elle marque donc le rapport entre passé, présent et futur. Par ailleurs, dans cette prestation formulée en terme monétaire, le 1, chiffre impair, signale une forme d'incomplétude qui est source de vie. En effet, tout chiffre pair est une forme de perfection, mais aussi de fermeture, de mort. On saisit donc ainsi l'importance du temps présent dans la construction de la relation de dette.

Dans le même mouvement qui consiste à rendre, à rembourser une dette, on ouvre une créance. Celui qui décide de rembourser une dette manifeste qu'il veut rompre la relation et nous n'avons pas eu beaucoup d'exemples de rupture de relations entre consanguins. Il n'y a pas de début ni de fin dans la relation de dette. Par rapport à un consanguin particulier, Ego est à un temps t1 débiteur, un temps $\mathrm{t} 2$ créancier, un temps $\mathrm{t} 3$ débiteur, etc. Mais aucun des moments considérés ne constitue le début de la dette, aucun autre n'en est la fin. Il n'y a donc pas d'origine d'une relation de dette ${ }^{7}$. L'arrêt n'est pas pensable globalement entre consanguins (même si certains peuvent être tentés de stopper cette relation de dette). La dette peut être remboursée lors d'un autre mariage, d'une circoncision ou de la sortie d'un deuil. À la mort d'un homme, c'est son fils aîné qui hérite des dettes qu'il a contractées. Autrement dit, la dette se perpétue dans la suite des générations. Les relations entre consanguins sont fondées sur la réciprocité entre égaux, mais les prestations renvoient aussi à des relations asymétriques de dettes. On peut même dire que ce qui constitue cette consanguinité et l'inscrit dans la durée, c'est d'une certaine manière cette relation de dette.

Il n'y a pas d'échange direct et immédiat. Au contraire, comme Pierre Bourdieu l'avait bien vu (1972: 221-222), l'attente est fondamentale dans ce type d'interrelation. Mais il ne s'agit pas ici d'affaire de stratégie, de rapport de rivalité, de pouvoir qui s'introduit dans l'échange par l'intermédiaire de la dette. Elle inscrit la relation de consanguinité dans la durée. En bref, c'est la relation de dette qui fonde l'échange et l'inscrit dans la diachronie. Dans ce cadre, il n'est pas besoin, contrairement à ce qu'avait pensé Mauss, d'évoquer «l'esprit de la chose donnée ". Ce qui est important dans la prestation, c'est la quantité de la somme : $5,11,51$, etc., et la manière de la répartir pour que l'échange se perpétue et se répète indéfiniment. La relation asymétrique entre débiteur et créancier peut et doit s'inverser et l'échange s'établir sur l'égalité des partenaires.

7. Une comparaison avec l'Inde brahmanique mérite d'être signalée ici. Charles Malamoud souligne à propos des termes sanscrits dans les textes védiques qu'ils n'ont pas d'étymologie et il ajoute : «... de même la dette congénitale de l'homme, si elle explique tout, ne s'explique par rien, et n'a pas d'origine. De même que la notion de dette est déjà là, toute formée, dans les plus anciens textes, de même la dette fondamentale affecte l'homme et le définit dès l'instant où il naît" (1989: 120). 


\section{Le don orienté de la femme}

À l'opposé, la relation entre affins est marquée par le don orienté de la femme et de la dot. Dans les deux cas, il est question de dan que nous avons traduit par don à la suite de Mauss (voir ci-dessus). On parle de kanya dan, "don de la jeune fille ", et de dan-dahej, "don de la dot ». Cette dot est constituée de divers objets pour la maison (lits, tables, ventilateur, etc.) pour le déplacement (vélo, moto et même parfois voiture), de vêtements, de bijoux, etc. C'est le père de la mariée qui donne sa fille en mariage et qui assume tous les frais qui sont souvent très importants. Le mari, accompagné par des membres de son groupe (lesquels sont appelés le barat) ainsi que d'un représentant de son père, vient chercher sa jeune épouse pour l'emmener, avec la dot, chez lui. Les Meo distinguent les preneurs et les donneurs et il n'est pas question d'inverser la relation avec l'un comme avec l'autre ${ }^{8}$. Dans la relation d'affinité qui s'établit et que la terminologie énonce clairement, entre un beau-père, un susar, une belle-mère, une sas, d'un côté, et un gendre, un asnaw, une bru, une bahu, il n'est pas question d'échange. Il est interdit de rendre une femme à celui à qui on en a pris ${ }^{9}$. Lors des cérémonies, le côté du marié peut certes apporter quelques cadeaux pour la mariée, mais cela est très inférieur à ce qu'il reçoit, et, de plus, il n'est pas question à ce

8. Les Meo sont endogames en ce sens qu'ils refusent de donner leurs femmes à l'extérieur de la caste ou de leur communauté. Chez les Meo, on ne peut pas se marier dans son clan, dans d'autres clans avec qui on dit avoir une parenté mythique, dans son village (qu'il soit composé d'un ou plusieurs groupes lignagers parents ou non). Les Meo reconnaissent comme unités matrimoniales pertinentes, les sous-clans pour les groupes territoriaux et les lignages pour les autres. Ils distinguent les preneurs, c'est-à-dire les groupes à qui ils donnent des femmes en mariage, des donneurs, c'est-à-dire les groupes qui leur donnent des épouses. Cette différence entre preneur et donneur est essentielle. Il n'y a aucun mariage par échange entre deux groupes et il n'est pas possible d'inverser l'orientation de l'alliance entre deux groupes donnés. Autrement dit, un groupe preneur ne donnera jamais ses femmes à celui qui lui a donné une épouse. Par ailleurs, l'alliance de mariage entre les groupes est transgénérationnelle, mais de manière particulière. On ne sait pas quand cette alliance a commencé ni quand elle se terminera. Tout mariage entre deux groupes est considéré comme perpétuant une alliance établie depuis un temps indéterminé pour ne pas dire immémorial.

9. Jonathan Parry décrit dans son ouvrage sur Kangra en Inde du Nord (1979) un exemple de mariage hypergamique avec différence de statut entre groupe donneur et groupe receveur sans possibilité d'échange ou d'inversion de la relation donneur-preneur. Dans cette société, il est difficile de trouver un époux pour le groupe du plus haut statut et une épouse pour un groupe du plus bas statut. Il existe des cas intermédiaires toujours en Inde du Nord comme l'a bien montré Véronique Pasche Huber (2002) à propos d'une caste de commerçants hindous du Rajasthan. Dans cette caste, il y a des mariages par échange de sœurs, ce qui semble contredire l'idéal du don orienté du type kanya dan auquel font référence explicitement les membres de cette caste. Mais outre que ce mariage est relativement mal considéré, il n'est pas répété et ne fonde pas une alliance de mariage de type diachronique. Par ailleurs, chaque mariage de cet échange est célébré séparément et la supériorité rituelle du preneur sur le donneur est affirmée comme dans le cas meo. Tout se passe comme si on cherchait à manifester la prééminence de l'asymétrie rituelle sur la réciprocité qui tout en étant pratiquée est dévalorisée (Pasche-Huber, 2002 : 435-441). Pour un cas similaire, voir T.N. Madan, 1965. 
propos de contre-don ou d'une quelconque réciprocité ${ }^{10}$. Donc ce que l'on marque, c'est bien l'orientation du don qui ne supporte aucun retour possible. Les Meo constituent un exemple parfait de la distinction nécessaire entre l'échange et le don.

\section{Les prestations du frère et le paiement rituel à la sœur}

Le don de la femme ne signifie pas que celle-ci est simplement un objet transmis ni même une valeur. Certes, son mariage l'associe dorénavant au groupe de son mari, mais cela ne signifie pas qu'elle cesse de faire partie de son groupe d'origine. Dans le village de son mari, elle est la bru et doit obéissance à sa belle famille. Cependant elle reste affiliée à son groupe d'origine. Sa double appartenance est bien marquée de plusieurs manières : ce n'est plus la relation d'affinité entre un beau-père et un gendre qui est opératoire dorénavant, mais la chaîne de relations frère-sœur comme on le verra plus bas. Comme la terminologie le montre bien dans la figure ci-dessous, un Ego masculin reconnaît deux bahin, deux « sœurs ", et un Ego féminin deux bhai, deux "frères » (avec cette spécificité que les termes de bahin-behnoi et bhai-bhabi renvoient à la fois à un couple mari-femme et à une paire frère-sœur).

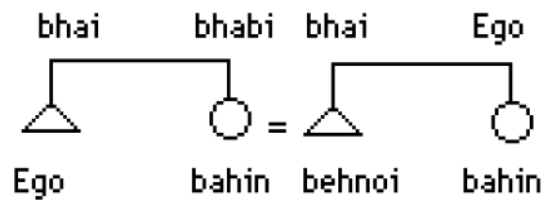

Ces deux termes réciproques constituent ce que j’ai appelé la métagermanité, en ce sens qu'ils définissent des relations qui transcendent la distinction entre consanguinité et affinité ${ }^{11}$. C'est au niveau rituel que cette chaîne de germains de sexe opposé liés par un mariage s'exprime pleinement. À partir du moment où une femme mariée a un enfant, son frère (oncle maternel des enfants), et pour qui elle est donc une bahin, une sœur, doit venir dans le village et le lignage du mari pour lui faire des prestations de différentes sortes (tissus, habits, bijoux, argent, etc.) : pour la naissance d'un fils, la circoncision d'un garçon, le mariage

10. Pour les Meo, les prestations vont toujours du donneur au preneur et cela, quelles que soient les circonstances de la vie, dans les moments profanes ou ordinaires comme dans les moments rituels. Un homme visitant sa sœur mariée reçoit certes un repas, mais il doit faire des dons à celle-ci comme à son mari et aux agnats de celui-ci au moment de son départ. Quand un homme en rencontre un autre, au marché, dans une ville ou lors d'une cérémonie, il doit s'enquérir pour savoir si celui-ci est dans la classe d'un consanguin ou d'un affin et dans ce dernier cas, s'il est du côté preneur ou donneur de femmes, s'il doit donner ou recevoir une prestation.

11. Pour plus de détails, voir le chapitre 2 : Le vocabulaire de parenté meo de mon ouvrage La relation frère-sour, parenté et rites chez les Meo de l'Inde du nord (1991 : 57-104). 
de ses enfants. C'est la sœur mariée qui se met en avant pour recevoir les biens et son mari se tient derrière elle ${ }^{12}$. Ces prestations sont destinées à elle, à ses enfants, mais aussi à la sœur du mari de sa sœur, c'est-à-dire l'autre bahin.

Plus encore, elle revient régulièrement là où elle est née, où elle est reçue avec beaucoup d'égards, et surtout, elle sera l'officiante principale des rituels de naissance, de circoncision des garçons et de mariage des enfants de son frère. Elle est dans ces circonstances appelée : sahvasani, littéralement « le compagnon femelle ${ }^{13}$. Son mari peut l'accompagner dans ces circonstances s'il le souhaite, mais il ne peut pas la remplacer dans ces rôles rituels. C'est généralement la sœur aînée (tante paternelle des enfants) qui occupe cette fonction, parfois une sœur cadette si elle fait preuve de charisme et de détermination. Si un homme n'a pas de sœur biologique, une sœur classificatoire sera choisie et aura la même fonction.

Cette position prééminente est considérée par certains comme l'équivalent de celle d'un brahmane chez les hindous. Lors de ces occasions rituelles, elle est la maitresse de la maison de son frère. Ses sœurs encore célibataires, ou ses sœurs mariées cadettes, la mère des enfants de son frère comme ce dernier doivent l'assister, l'honorer et lui obéir. En bref, tout indique que cette officiante, cette sahvasani est donc la sour telle que le mariage l'a transformée.

Cette sœur mariée consacre, lors de différentes cérémonies, la maison de son frère (laquelle est blanchie, les ustensiles de cuisine sont renouvelés, etc.) les futurs mariés (ils sont baignés, purifiés, sont l'objet de nombreux interdits notamment alimentaires etc.) Cette action rituelle, qui élève ce futur couple à une dignité royale, est celle qui rend possible le don de la jeune fille et sa réception chez le marié. Pour ses services rituels, elle reçoit un paiement appelé neg. En aucune manière, il ne s'agit ici d'un don ou d'un échange, mais d'honoraires pour le travail rituel qu'elle a effectué. De ce point de vue, le versement du neg dans le mariage est à rapprocher du payement de la daksina à l'officiant dans le sacrifice (voir plus bas). En bref, la femme mariée se transforme : d'objet du don, elle devient un sujet actif comme sœur qui officie pour les enfants de son frère et qui reçoit de ce dernier une rétribution pour ses services. Dans ce contexte, la sœur occupe une position prééminente par rapport à son frère. Par ailleurs, si le frère reçoit les services rituels de sa sœur, il ne peut pas rendre la pareille pour les enfants de cette dernière. Il n'y a donc pas de réciprocité, mais bien orientation comme avec le don.

12. S. Vatuk a très bien analysé ce problème. En effet, après avoir souligné que la relation donneur-preneur suppose des dons "perpétuels ", elle ajoute: "This relationship is mediated through the person of the 'bride' herself, who not only serves (qua bride) as the supreme gift in and of herself, but also serves(qua daughter or sister) as the key recipient of gifts" (Vatuk, $1975:$ 174).

13. P. Aggarwal qui a étudié les Meo souligne aussi l'importance du rôle rituel de la sahvasani (1971: 180 ssq.) 


\section{Le service rituel dans la perspective diachronique}

Nous avons souligné à propos de l'échange-dette entre consanguins leur répétition et leur inscription dans la diachronie. Il faut souligner ce qu'il en est du don et du paiement rituel dans l'enchaînement diachronique des mariages. Nous avons fait jusque-là comme si les interventions de la sœur mariée comme officiante et les prestations du frère à sa sœur étaient effectuées au bout de la chaîne, après le don, comme le montre le schéma suivant :

Don (Do) $\rightarrow$ Prestations (Pr) / Service et paiement

rituel $(\mathrm{SPa})$

Affinité $(\mathrm{A}) \rightarrow$ Relation frère/sœur $(\mathrm{F} / \mathrm{S})$

En fait, comme on peut le constater, les choses sont plus compliquées. Les cérémonies de mariage ne commencent qu'après l'arrivée du côté du garçon comme du côté de la fille, de la tante paternelle qui va officier et après les prestations cérémonielles de l'oncle maternel. Ce n'est qu'après que commencent les rapports d'affinité avec le don de la jeune fille, etc. On peut donc établir le schéma suivant :

$\mathrm{Pr} / \mathrm{SPa}$ (génération 1) $\rightarrow$ Do $\rightarrow \mathrm{Pr} / \mathrm{SPa}$ (génération 2)

$\mathrm{F} / \mathrm{S}$ (génération 1) $\rightarrow \mathrm{A} \rightarrow \mathrm{F} / \mathrm{S}$ (génération 2)

qui renvoie à la figure ci-dessous :

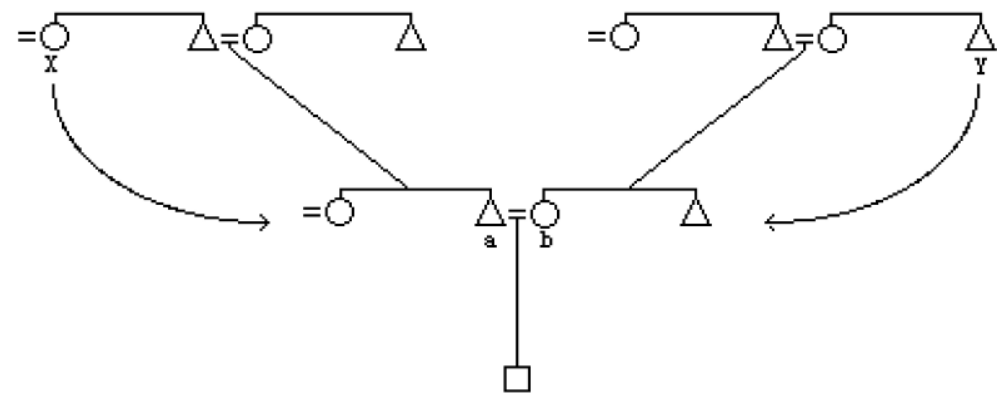

Dans un premier temps donc, une double paire frère-sœur (à quoi correspondent $\mathrm{Pr} / \mathrm{SPa} 1$ et $\mathrm{F} / \mathrm{S} 1$ ) intervient de chaque côté. Mais après avoir officié pour les enfants de son frère, la sœur mariée, la tante paternelle pour les enfants a et b, cesse d'intervenir. Elle sera encore plus associée au lignage de son mari et sera enterrée dans le même cimetière que lui. L'oncle maternel des enfants a et b n'est plus dans l'obligation de donner à la génération suivante. On peut donc dire que le paiement rituel fait à la sœur mariée est le processus qui conduit à terme à la séparation entre le frère et la sœur.

Mais, en agissant ainsi, la sœur mariée ouvre un nouveau cycle de dettes entre consanguins, de don de la jeune fille à des affins. Une nouvelle génération prend la suite (F/S2 et $\mathrm{Pr} / \mathrm{SPa} 2)$. Le frère de cette femme b succédera à son oncle maternel ( $\mathrm{Y}$ sur le schéma) et fera les mêmes prestations que celui-ci, pour le 
mariage des enfants de sa sœur. Une nouvelle sœur mariée qui succède à sa tante paternelle (X sur le schéma) viendra chez son frère pour officier, notamment lors des mariages de ses enfants. La figure ci-dessus montre comment la nièce succède à sa tante paternelle côté garçon et le neveu à son oncle maternel côté fille.

La suite des générations ne peut s'effectuer sans l'intervention de cette double relation frère-sœur liée par un mariage, et donc par l'ensemble prestations cérémonielles d'un côté et service/paiement rituel de l'autre. Ce qui conclut un rituel de mariage en engage un autre et il n'y a pas de fin à cet enchaînement de cycles.

\section{Mariage et sacrifice}

J. Parry a signalé l'homologie entre l'analyse du don orienté et celle du sacrifice (voir plus haut). Cette remarque mérite d'être prise en considération et nous suivrons ici certains points des travaux de Charles Malamoud. La comparaison entre les cérémonies de mariage meo et le sacrifice védique doit éviter plusieurs fausses pistes. Il ne s'agit pas de chercher dans le sacrifice védique d'il y a plus de deux mille ans une quelconque norme par rapport à laquelle il faut analyser les rites de la période contemporaine. Il ne s'agit pas non plus de comparer élément par élément les deux ensembles de rites qui sont très différents et sont donc incomparables de ce point de vue. Notre propos est de nous intéresser à certains principes régissant les rituels sacrificiels comme les cérémonies de mariage, pour apporter un éclairage à notre analyse Deux homologies ont retenu notre attention :

- Les deux types de rituel commencent par une consécration, celle du sacrifiant et de la victime dans le sacrifice et celle des futurs époux et de leur parents respectifs. C'est à cette condition que le rapprochement entre l'offrande sacrificielle et le don de la femme est fait. La référence au sacrifice nécessite néanmoins une explication. Malamoud a montré que le sacrifice védique a servi de modèle pour interpréter toute manifestation rituelle, notamment les rites des âges de la vie, comme le mariage ou les funérailles. Certes, depuis plus de deux mille ans, le sacrifice en tant que tel n'est plus la manifestation principale des faits rituels indiens. Mais une certaine continuité persiste au niveau du sacrifice comme modèle, comme processus de transformation rituelle. À ce propos, Malamoud souligne que les textes védiques considèrent le mariage de deux manières : soit comme un samskara, un "perfectionnement ", c'est-à-dire comme un rite de passage pour l'homme et la femme ; soit comme un yajna, un "sacrifice " mettant en relation les différents partenaires de la cérémonie. "Dans ce yajna, le rôle du sacrifiant est tenu par le père de la fille ; celle-ci est la victime, pasu; et le jeune homme, destinataire de cette offrande, a donc la fonction de devata (divinité) ; quant aux rtvij (officiants), leur fonction est remplie par les divers purohita présents à la cérémonie » (Malamoud, 1974-1975 : 124). Dans la cérémonie meo, nous retrouvons le père qui donne sa fille, le mari qui la reçoit, et 
l'épouse comme don, similaire à l'offrande sacrificielle. Dans les deux cas, l'officiant, le purohita et la sœur mariée (devenue tante paternelle), conduisent le rituel et rendent possible respectivement l'offrande et le don. Dans les deux cas, il y a une asymétrie entre les deux côtés : le preneur (assimilé à la divinité dans le sacrifice) étant supérieur au donneur (assimilé au sacrifiant), le don de la fille constituant comme l'offrande, le lien entre les deux côtés. En bref, le don orienté loin d'être inclus dans l'échange, doit plutôt être rapproché de l'offrande sacrificielle. Dans les deux types de rituels, le mécanisme qui est mis en œuvre nécessite un ensemble de relations sociales orientées et hiérarchisées.

- On peut établir une seconde homologie entre le travail accompli par une sœur mariée pour son frère et les enfants de celui-ci et le travail décrit dans le sacrifice védique. Dans son article : "Terminer le sacrifice. Remarques sur les honoraires rituels dans le brahmanisme » (1976), Charles Malamoud souligne l'importance de cette relation entre le sacrifiant et l'officiant. Le premier est consacré par le second et quitte un corps profane pour acquérir un corps divin. L'officiant (le brahmane dans le sacrifice) permet cette transformation et accompagne le sacrifiant dans les différentes opérations rituelles. Il reçoit une rétribution, la daksina, pour le service rituel qu'il a accompli ; Malamoud remarque que la daksina n'est pas une étape mineure du sacrifice, mais constitue un moment important du rituel. Elle n'est pas un contre don en réponse au don comme le pensent certains sanscritistes. C'est un honoraire rituel, une rémunération analogue à celui fait dans le cadre de la vie économique. Voici ce que le texte védique souligne : "Il faut considérer que le daksinâ a pour fonction de "louer" (les services) parce qu'il fait mention de tâches, tout comme dans la vie courante. » "Dans la vie courante aussi, quand on donne quelque chose à un porteur de bois, à un travailleur quelconque, c'est pour louer leur services, même s'il n'est pas dit explicitement que c'est une location de service qu'il s'agit »... Les biens donnés aux officiants sont une rémunération parce que leur activité est un travail... «de même que le bûcheron qui coupe du bois, quand il est engagé pour ce travail, se met à ce travail, de même les prêtres officiants de ce sacrifice " (1976:181-182). Malamoud ajoute : "Ce qui frappe... c'est que la daksina, si elle n'est pas un salaire, sert de modèle au salaire » (ibid. : 183). L'homologie avec les cérémonies de mariage est frappante de ce point de vue : dans les deux cas, le rituel nécessite une consécration, un abandon du corps profane pour endosser un corps sacré ; dans les deux cas, cela n'est possible que grâce au travail rituel effectué par un officiant. La sœur mariée dans un cas et le brahmane dans l'autre ; enfin le paiement du neg est similaire à celui de la daksina. Dans les deux cas, il s'agit d'une rémunération pour service rendu et non d'un contre-don en réponse à un don.

Charles Malamoud souligne que payer l'officiant constitue l'acte conclusif du sacrifice. Il permet au sacrifiant de se reprendre après s'être donné et de mettre l'officiant à distance. "Le prix à payer pour reprendre son corps au rtvij, c'est la daksina " (ibid. : 193). Alors que la consécration et l'offrande constituent l'entrée dans le rite sacrificiel, le paiement des honoraires constitue la sortie du rituel. De la même manière, dans les cérémonies du mariage, le don de la jeune 
fille consacrée par l'officiante marque l'entrée et le moment central du rite, alors que le paiement du neg à cette officiante en est la conclusion. Le don et le service pour lequel il y a un paiement relèvent certes de deux types de prestations différents, mais ils s'agencent dans le même mécanisme rituel.

\section{Conclusion : pour une analyse rituelle de la parenté}

Nous sommes partis d'une remarque de Marcel Mauss sur le don indien qui interdit tout retour, tout contre-don. Cela nous a amenés à questionner la généralisation du lien entre don et échange aussi bien dans la circulation des biens que dans l'alliance du mariage. Rappelons que dans son analyse du hau maori, Mauss souligne : " c'est que la chose donnée n'est pas inerte. Même abandonnée par le donateur, elle est encore quelque chose de lui. Par elle a prise sur le bénéficiaire " (Mauss, 1960 : 159). Nous avons vu que la sœur donnée en mariage garde son affiliation avec son groupe d'origine. Mais si la remarque de Mauss s'applique à l'exemple indien des Meo, la nécessité de rendre et donc d'échanger des femmes ne nous paraît pas s'imposer contrairement à ce qu'il a affirmé. Entre le don gratuit et le don qui nécessite un contre-don, il faut donc situer le don orienté obligatoire. Le retour de la sœur mariée est celui d'une officiante et de ce point de vue, c'est le rapprochement du don avec le mécanisme sacrificiel qui s'impose.

L'analyse ethnographique du cas des Meo de l'Inde du Nord nous conduit donc à prendre en compte le rapport entre don rituel, mécanisme sacrificiel et alliance de mariage. Ce sont les travaux fondateurs d'Hubert et Mauss sur le sacrifice, de Mauss sur le don, de Lévi-Strauss sur le mariage, qui ont construit de manière originale et très semblable ces trois objets ethnologiques. Ceux-ci ont connu des développements et des questionnements méthodologiques et théoriques importants et il n'est pas dans notre intention ici d'y revenir ${ }^{14}$. On notera le problème de leur agencement. Claude Lévi-Strauss a bien utilisé le travail sur le don pour son analyse des structures élémentaires de la parenté, mais c'est pour en faire une manifestation des échanges et comme on l'a souligné plus haut, l'archétype des échanges. Dans la conclusion de leur étude sur le sacrifice, Hubert et Mauss soulignent que le don se trouve en rapport avec le sacrifice. Comme ils l'écrivent : "... on a pu voir, chemin faisant, combien de croyances et de pratiques sociales, qui ne sont pas proprement religieuses, se trouvent en rapport avec le sacrifice. Il a été successivement question du contrat, du rachat, de la peine, du don, de l'abnégation" (p. 307). À l'inverse, dans l'Essai sur le don, il est indiqué que le don peut être considéré sous l'angle du sacrifice : « La destruction sacrificielle a précisément pour but d'être une donation qui soit nécessairement rendue. Toutes les formes du potlatch du nord-ouest américain et nord-

14. Un article en préparation soulignera les principaux développements sur ces différents objets ethnologiques. 
est asiatique connaissent ce thème de la destruction. Ce n'est pas seulement pour manifester puissances et richesses et désintéressement qu'on met à mort des esclaves, qu'on brûle des huiles précieuses, qu'on jette des cuivres à la mer, qu'on met même le feu à des maisons princières. C'est pour sacrifier aux esprits et aux dieux, en fait confondus avec leur incarnation vivante, les porteurs de leurs titres, leurs alliés initiés»(p. 167). Mais il ne s'agit que d'indications qui n'ont pas connu de développement. La recherche a continué sur la spécificité de chaque objet. Notre analyse nous a amenés au contraire à insister sur l'interrelation entre le mariage, le don et le mécanisme sacrificiel en les séparant de l'échange qui est fonction de la dette. Mais cette nouvelle approche n'a de sens que si ces objets sont considérés dans le contexte rituel où ils se déploient comme nous l'avons montré dans le cas de l'ethnographie meo : l'échange et le don relèvent de deux domaines de la parenté qui sont liés: la consanguinité pour l'un et l'affinité pour l'autre, mais cette complémentarité ne se manifeste que dans les cérémonies de mariage. De même, la relation entre le don et le sacrifice ne prend sens que si l'on considère le travail rituel comme un service fait par un officiant pour lequel il reçoit un paiement, toujours dans ces mêmes cérémonies. L'homologie que nous avons signalée du don de la femme comme offrande sacrificielle et celle entre le neg dans le mariage et la daksina dans le sacrifice védique est à situer dans ce contexte où, comme on l'a dit, l'on compare non pas les éléments du sacrifice et ceux des cérémonies du mariage, lesquels sont incomparables, mais les principes qui sont à l'œuvre dans le processus rituel.

Les travaux ethnologiques sur le mariage ont insisté depuis Lévi-Strauss, sur le choix du conjoint et ses implications. La question des prestations cérémonielles au sens large a été considérée soit comme relevant d'un autre domaine, soit étudiée comme le prolongement du choix matrimonial. Je rappellerai ce qui a été signalé plus haut : la position de Lévi-Strauss sur l'union matrimoniale comme archétype de l'échange, et celle de Dumont qui certes a insisté sur les prestations cérémonielles à propos de la parenté dravidienne des Pramallai Kallar (Inde du Sud), mais son analyse ne faisant que confirmer la primauté du choix du conjoint, le mariage avec la cousine croisée matrilatérale. Notre analyse du cas méo nous a amené à changer ce type de perspective. C'est dans le rituel que se manifestent les différents types de prestations : l'échange, le don orienté et le paiement des honoraires. Si l'analyse du cas meo a une valeur, c'est de nous amener à questionner une analyse de la parenté comme réalité sui generis qui a son autonomie et plus largement à penser que la théorie de la parenté ne peut pas faire l'économie des formes diversifiées des prestations cérémonielles et donc de la logique rituelle proprement dite.

Raymond JAMOUS

CNRS, Laboratoire d'ethnologie et de sociologie comparative raymond.jamous@mae.u-paris10.fr 
I96 - ARCHIVES DE SCIENCES SOCIALES DES RELIGIONS

\section{Bibliographie}

Aggarwal Partap, 1971, Caste, Religion and Power, New Delhi, Shri Ram Centre for Industrial Relations.

BouRdieu Pierre, 1972, Esquisse d'une théorie de la pratique, Genève, Librairie Droz.

Dumont Louis, 1957, Une sous-caste de l'Inde du Sud. Organisation sociale et religion des Pramalai Kallar. Paris-La Haye, Mouton. [Réimpr. Paris, Éditions de l'EHESS, 1992]

-, 1966 « Marriage in India. The present state of the question: III North India in relation to South India ", Contribution to Indian Sociology, 9, pp. 90-114.

-, 1971 Introduction à deux théories d'anthropologie sociale. Groupes de filiation et alliance de mariage, Paris-La Haye, Mouton-EPHE, $\mathrm{VI}^{\mathrm{e}}$ section, coll. "Les textes sociologiques », 6 .

Hubert Henri, Mauss Marcel, 1968, "Essai sur la nature et la fonction du sacrifice ", in Mauss M., Euvres, 1. Les fonctions sociales du sacré, pp. 193-307.

JAMOUS Raymond, 1981, Honneur et Baraka. Les structures sociales traditionnelles dans le Rif, Cambridge - Paris, Cambridge University Press - Maison des Sciences de l'Homme.

-, 1991 La relation frère-sour. Parenté et rites chez les Meo de l'Inde du Nord, Paris, Éditions de l'EHESS.

LÉvi-Strauss Claude, 1960, "Introduction », in Mauss M., Sociologie et Anthropologie, Paris, Presses Universitaires de France, IX-LII.

-, 1962, La pensée sauvage, Paris, Plon.

-, 1967, Les structures élémentaires de la parenté, Paris, Mouton.

Madan T. N., 1965, Family and Kinship. A study of the Pandits of Rural Kashmir, New Delhi, Oxford University Press.

Malamoud Charles, 1974-1975, "Résumé des conférences et travaux ", in Annuaire de l'École Pratique des Hautes Études - Ve section, pp. 122-127.

-, 1976, "Terminer le sacrifice. Remarques sur les honoraires rituels dans le brahmanisme ", in Biardeau M., Malamoud Ch., (éds.), Le sacrifice dans l'Inde ancienne, Paris, Presses Universitaires de France, pp. 155-204.

Mauss Marcel, 1960, "Essai sur le don, forme et raison de l'échange dans les sociétés archaïques ", in Mauss M., Sociologie et Anthropologie, Paris, Presses Universitaires de France, pp. 145-279.

PARry Jonathan, 1979, Caste and Kinship in Kangra, London, Routledge \& Kegan Paul. -, 1986 "The gift, the Indian Gift and the "Indian Gift" ", Man, NS 21-3, pp. 453-473.

-, 1994, Death in Banares, Cambridge, Cambridge University Press.

Pasche-Huber Véronique, 2002, Noces et négoces. Dynamiques associatives d'une caste de commerçants hindous, Neuchâtel - Paris, Éditions de l'Institut d'ethnologie - Éditions de la Maison des sciences de l'homme.

Raheja Gloria Goodwin, 1988, The Poison in the Gift, Ritual, Prestation, and the Dominant Caste in a North Indian Village, Chicago, Chicago University Press.

ReINICHE Marie-Louise, 1977, "La notion de "Jajmânî". Une qualification abusive ou principe d'intégration ", Purusârtha, III, pp. 71-107.

Testart Alain, 2007, Critique du don. Études sur la circulation non marchande, Paris, Éditions Syllepse.

Trautmann Thomas R., 1981, Dravidian Kinship, Cambridge, Cambridge University Press. 
Vatuk Sylvia, 1969, "A Structural of the Hindi Kinship Terminology ", Contributions to Indian Sociology, NS 3, pp. 94-115.

-, 1975, "Gifts and Affines in North India ", Contribution to Indian Sociology, NS 9-2, pp. 155-196.

\section{Résumé}

Cette étude confronte trois "objets ethnologiques", le don rituel, le sacrifice et le mariage à des modèles - ceux de Lévi-Strauss, de Mauss ou de Dumont - qui dissocient ou qui, au contraire, interpénètrent ces objets dans la construction de leur système d'interprétation. C'est par cette voie, empirique et théorique, que Raymond Jamous compose une définition du mariage du point de vue de la désignation des conjoints et des implications de l'alliance à une approche rituelle qui conduit l'auteur à discuter l'échange comme principe essentiel ou unique de l'alliance, au bénéfice d'une autre conception d'un don sans contre-don, qui à son tour articule la logique rituelle sur une logique sacrificielle. L'analyse du cas Méo, caste de guerriers $d u$ Rajasthan, vérifie cette étroite imbrication du don rituel, du mécanisme sacrificiel et de l'alliance matrimoniale, dans une perspective qui conduit une discussion concrète avec le classique Essai sur le don et fait apparaître la force du moment cérémonial dans une anthropologie de la parenté.

Mots-clés : objets ethnologiques, mariage, don, castes, parenté.

\section{Resumen}

Este estudio confronta tres "objetos etnológicos", el don ritual, el sacrificio y el matrimonio, a modelos -los de Levi-Strauss, de Mauss o de Dumont-que disocian o que por el contrario interpenetran estos objetos en la construcción de su sistema de interpretación. Por esta vía, empírica y teórica, Raymond Jamous elabora una definición del matrimonio desde el punto de vista de la designación de los cónyuges $y$ de las implicaciones de la alianza, en un abordaje ritual que conduce al autor a discutir el intercambio como principio esencial o único de la alianza, en beneficio de otra concepción de un don sin contra-don que, a su vez, articula la lógica ritual sobre una lógica sacrificial. El análisis del caso Meo, casta de guerreros de Rajastán, verifica esta estrecha imbricación del don ritual, del mecanismo sacrificial y de la alianza matrimonial, en una perspectiva que lleva una discusión concreta con el clásico Ensayo sobre el don, y hace aparecer la fuerza del momento ceremonial en una antropología del parentesco.

Palabras clave: objetos etnológicos, matrimonio, don, castas, parentesco. 
